\title{
Impact of Diagnosis-Related Group Payment System on the Variation in Hospitalization Expenditures: A Case-Control Study with a Propensity-Score-Matched Control Group
}

\section{Xiaoqi Fan}

Peking University Health Science Center

\section{Zhifan Wang}

Peking University Health Science Center

\section{Shanshan Huo}

Peking University Health Science Center

\section{Ziyan Chen}

Peking University Health Science Center

Weiyan Jian ( $\sim$ jianweiyan@bjmu.edu.cn )

Peking University Health Science Center

\section{Research Article}

Keywords: diagnosis-related group, payment reform, health services, variation in hospitalization expenditures

Posted Date: December 10th, 2021

DOI: https://doi.org/10.21203/rs.3.rs-1131573/v1

License: (c) (i) This work is licensed under a Creative Commons Attribution 4.0 International License. Read Full License 


\section{Abstract \\ Background}

One of the important ways to reduce medical costs and improve quality of care is to enable physicians to provide standard medical services according to clinical guidelines, and the medical payment system is a significant means of guiding the behaviour of health service providers. This study aims to investigate whether the diagnosis-related group (DRG) payment system can improve the consistency of health services.

\section{Method}

Inpatients with three types of disease-chronic obstructive pulmonary disease (COPD), acute myocardial infarction (AMI) and cerebral infarction (Cl)-were enrolled from 25 county-level hospitals in a DRG pilot city in China. Inpatients from hospitals that implemented DRG payment were selected as the intervention group, and similar inpatient cases from hospitals that still implemented fee-for-services (FFS) payment were designated as the control group. A propensity matching score (PSM) was used for data matching to control for age, gender and disease severity. The variation of hospitalization expenditures and their trends before and after implementation of the DRG policy were described by using these matched samples.

\section{Results}

After DRG implementation, the standard deviation (SD) of hospitalization expenditures in the COPD, AMI and $\mathrm{Cl}$ intervention groups decreased by 11094 yuan, 4833 yuan and 425 yuan, respectively, which were 5972, 2484, and 2938 yuan more than that in the control group. In each year after DRG implementation, the interquartile range (IQR) of hospitalization expenditures was smaller in DRG group than that in FFS group. In most years, the degree of variation in costs of the intervention group decreased more than that of the control group. The medians of hospitalization expenditures of the intervention groups were lower than the fixed cost, while most medians of the control groups were higher than the fixed cost.

\section{Conclusion}

A comparison of patients with similar demographics and disease characteristics revealed that patients in the DRG group experienced a smaller degree of variation in hospitalization expenditures, and indicated the expenditures had a tendency to become progressively more concentrated over time. It is suggested that DRG system can promote better consistency in health services and reduce medical costs.

\section{Background}


Many countries are concerned about the variation in health services, which is not only related to the control of medical costs but also to the guarantee of quality of care. The relationship between the cost and quality of health services is multifaceted. On the one hand, efforts to improve the quality of care will increase the medical costs $(1,2)$, and the difference of medical costs at different levels of quality of care will also increases. On the other hand, with the general improvement in quality of care, complications and comorbidities will be mitigated, thus reducing medical costs (3), and the degree of variation between the costs will also decline. In fact, the relationship between cost and quality depends on where doctors intend to spend the money (4). Therefore, the general consensus of these studies is that doctors' practices play a crucial role in the production of these variations $(5,6)$.

It is the common hope of patients and even medical insurers that health service providers can provide standard services according to clinical guidelines, which can also improve the consistency of services and reduce the unnecessary waste. The medical payment system is an important means to guide the behaviour of health service providers. However, there is little literature on whether the reform of payment system can improve the consistency of health services.

The philosophy of health service consistency and DRG payment reform is particularly relevant. This is due to the similarity in clinical process and resource consumption among patients in the same DRG group (6).. For each DRG group, the hospitals are paid based on a flat rate rather than the actual medical costs. Therefore, after DRG payment, health service providers are more motivated to reduce inpatients' cost overruns (7). The induced demand is suppressed, and doctors are more motivated to implement the treatment according to the clinical guidelines (8). Thus, the DRG policy incentivizes hospitals to rationalize the diagnosis and treatment process (9). With DRG payment, treatment for similar patients will be more consistent than those paid by FFS.

DRG payment reform has been piloted in China, and many experiences show that the implementation of DRG shortened the length of hospital stay and controlled the costs (8). However, whether doctors' practices are more normative, the difference between medical and surgical costs and the impacts of disease severity on the difference remain to be measured. This study analyses the trends of the variation in hospitalization expenditures among patients with same diseases and different payment methods, attempts to fill the knowledge gap between payment reform and health service consistency, and to provide evidence to support the improvement of DRG payment reform.

\section{Methods}

\subsection{Study design}

Discharge data for beneficiaries of the Urban Employee Basic Medical Insurance were used to analyse reductions in the variation of hospitalization expenditures in a DRG pilot city of China. In 2012, the payment method began to shift from FFS payment to DRG payment in some hospitals, which served as intervention groups in this study. To generate the best comparison, we ensured that the types of patients, 
the number of beds, the hospital grades and the doctors in the control hospitals that still implemented FFS payment were similar to those in the intervention hospitals. The zero-markup drug policy in $2016 \mathrm{had}$ a great impact on the hospitalization expenditures in both intervention and control of hospitals.

Therefore, to ensure accurate measurement of the impacts of DRG payment, the study only observed the patient data from 2010 to 2016.

\subsection{Selection of participants}

To avoid the influence of outliers, the study limited the sample to inpatients whose hospitalization days were less than or equal to 60 days and whose total cost was more than 0 yuan. Patients with AMI were selected as representatives of the surgical group, and patients with either COPD or $\mathrm{Cl}$ were selected as representatives of the internal medicine group. The main diagnostic codes for $\mathrm{AMI}, \mathrm{Cl}$ and COPD started with I21.0, 163.0 and J44.0, respectively. The severity of the disease was assessed by the Charlson Comorbidity Index $(\mathrm{CCl})$ based on secondary diagnoses. All cost data were adjusted for inflation using the city's consumer price index (CPI) from the official website of the China Bureau of Statistics.

\subsection{Statistical analysis}

The intervention group and the control group were matched by 1:1 PSM year after year. To exclude the influence of individual differences on the outcome, the propensity score was obtained by logistic regression of the patients' age, gender, and $\mathrm{CCl}$. The Caliper value of the matching accuracy was 0.02 . The policy was implemented in 2012, so the patients in the intervention group before 2012 were matched with the patients in the intervention group after PSM matching in 2012, and then the patients in the control group before 2012 were matched.

The medical costs varied significantly among the different DRG groups. To further ensure the comparability and the application scope of the study, the DRG group with the largest number of patients with the same disease was selected for analysis. The DRG group of patients with COPD was defined as "COPD without complications and concomitant disease group", the DRG group of patients with AMI was defined as "coronary drug-eluting stent implantation with important complications group", and the DRG group of patients with $\mathrm{Cl}$ was defined as "cerebral ischaemic diseases with important complications and concomitant diseases group". The study compared the change trends of hospitalization expenditures and its variation between the intervention group and the control group before and after the implementation of DRG payment.

\section{Results}

\subsection{Basic information}

From 2010 to 2016, 14,094, 17,897 and 26,063 patients with COPD, AMI and Cl were assessed by the DRG grouping apparatus, respectively. After PSM matching, 6,238, 11,086, and 14,906 patients with COPD, $\mathrm{AMI}$, and $\mathrm{Cl}$ were enrolled in the study respectively, with half in the control group and half in the intervention group. 
Table 1 shows the sociodemographic characteristics of patients in the control and intervention groups by disease type after PSM matching, including the number of patients by age, gender, and disease severity. In COPD patients, the number of males was twice more than that of females, more than $80 \%$ patients were over 59 years old, and more than $80 \%$ patients had less severe diseases. In AMI patients, the number of males was four times more than that of females, more than $90 \%$ patients were aged 40 to 79 , and $82 \%$ patients had less severe diseases. In Cl patients, the number of males was approximately three times of females, more than $80 \%$ patients were aged 40 to 79 , and more than $50 \%$ patients had severe diseases. After matching, there was no significant difference in the distribution of age, gender or disease severity between the control and the intervention groups. 
Table 1

Sociodemographic characteristics

\begin{tabular}{|c|c|c|c|c|c|c|}
\hline \multirow[t]{2}{*}{ Diseases } & \multicolumn{2}{|c|}{ Variables } & \multicolumn{2}{|c|}{ Control Group } & \multicolumn{2}{|c|}{ Intervention Group } \\
\hline & & & $\mathrm{N}$ & $\%$ & $\mathrm{~N}$ & $\%$ \\
\hline \multirow[t]{10}{*}{ COPD } & \multirow[t]{2}{*}{ Gender } & Male & 2172 & 69.64 & 2160 & 69.25 \\
\hline & & Female & 947 & 30.36 & 959 & 30.75 \\
\hline & \multirow[t]{4}{*}{ Age } & $18-39$ & 3 & 0.10 & 2 & 0.06 \\
\hline & & $40-59$ & 329 & 10.55 & 340 & 10.90 \\
\hline & & $60-79$ & 1966 & 63.03 & 1983 & 63.58 \\
\hline & & $\geq 80$ & 821 & 26.32 & 794 & 25.46 \\
\hline & \multirow[t]{3}{*}{$\mathrm{CCl}$} & 0 & 1279 & 41.01 & 1245 & 39.92 \\
\hline & & 1 & 1300 & 41.68 & 1311 & 42.03 \\
\hline & & $\geq 2$ & 540 & 17.31 & 563 & 18.05 \\
\hline & \multicolumn{2}{|l|}{ Count } & 3119 & 100 & 3119 & 100 \\
\hline \multirow[t]{10}{*}{ AMI } & \multirow[t]{2}{*}{ Gender } & Male & 4461 & 80.48 & 4456 & 80.39 \\
\hline & & Female & 1082 & 19.52 & 1087 & 19.61 \\
\hline & \multirow[t]{4}{*}{ Age } & $18-39$ & 130 & 2.35 & 162 & 2.92 \\
\hline & & $40-59$ & 2450 & 44.20 & 2474 & 44.63 \\
\hline & & $60-79$ & 2633 & 47.50 & 2587 & 46.67 \\
\hline & & $\geq 80$ & 330 & 5.95 & 320 & 5.77 \\
\hline & \multirow[t]{3}{*}{$\mathrm{CCl}$} & 0 & 2335 & 42.13 & 2293 & 41.37 \\
\hline & & 1 & 2252 & 40.63 & 2286 & 41.24 \\
\hline & & $\geq 2$ & 956 & 17.25 & 964 & 17.39 \\
\hline & \multicolumn{2}{|l|}{ Count } & 5543 & 100 & 5543 & 100 \\
\hline \multirow[t]{6}{*}{$\mathrm{Cl}$} & \multirow[t]{2}{*}{ Gender } & Male & 5538 & 74.31 & 5447 & 73.08 \\
\hline & & Female & 1915 & 25.69 & 2006 & 26.92 \\
\hline & \multirow[t]{4}{*}{ Age } & $18-39$ & 161 & 2.16 & 151 & 2.03 \\
\hline & & $40-59$ & 2531 & 33.96 & 2527 & 33.91 \\
\hline & & $60-79$ & 3977 & 53.36 & 3994 & 53.59 \\
\hline & & $\geq 80$ & 784 & 10.52 & 781 & 10.48 \\
\hline
\end{tabular}




\begin{tabular}{|llllll|} 
CCI & 0 & 649 & 8.71 & 403 & 5.41 \\
\cline { 2 - 6 } & 1 & 3062 & 41.08 & 3225 & 43.27 \\
\cline { 2 - 6 } & $\geq 2$ & 3742 & 50.21 & 3825 & 51.32 \\
\hline Count & & 7453 & 100 & 7453 & 100 \\
\hline
\end{tabular}

Table 2 shows the composition and proportion of hospitalization expenditure in the intervention group and the control group by disease type, including drug expenditure, medical material expenditure, other hospitalization expenditure and their proportions respectively. In patients with COPD and $\mathrm{Cl}$, the drug expenditure was the highest, accounting for approximately $50 \%$, and the medical material expenditure was the lowest, accounting for approximately $10 \%$. The expenditure of medical material was the main expense for AMI patients, accounting for more than $70 \%$. After matching, there was no significant difference in the distribution of health expenditures between the control and the intervention groups.

Table 2

Composition of hospitalization expenditure of patients

\begin{tabular}{|llllllll|}
\hline \multirow{2}{*}{ Diseases } & Group & \multicolumn{2}{c}{ Drug Expenditure } & \multicolumn{2}{c|}{ Material Expenditure } & \multicolumn{2}{c|}{ Other Expenditure } \\
\cline { 3 - 8 } & & Yuan & $\%$ & Yuan & $\%$ & Yuan & $\%$ \\
\hline \multirow{2}{*}{ COPD } & Control Group & 7937.14 & 52.05 & 1513.26 & 9.92 & 5798.31 & 38.02 \\
\cline { 2 - 8 } & Intervention Group & 7423.55 & 52.47 & 1459.50 & 10.32 & 5264.79 & 37.21 \\
\hline \multirow{2}{*}{ AMI } & Control Group & 5386.27 & 8.55 & 46355.30 & 73.58 & 11254.61 & 17.87 \\
\cline { 2 - 8 } & Intervention Group & 4649.69 & 8.51 & 39928.32 & 73.09 & 10052.18 & 18.40 \\
\hline Cl & Control Group & 9550.41 & 49.18 & 2012.43 & 10.36 & 7854.91 & 40.45 \\
\cline { 2 - 8 } & Intervention Group & 7184.32 & 50.41 & 1221.47 & 8.57 & 5846.70 & 41.02 \\
\hline
\end{tabular}

\subsection{Main results for patients with COPD}

Table 3 and Figure 1 show the variation in hospitalization expenditures of COPD patients in two dimensions, standard deviation (SD) and interquartile range (IQR), respectively. The "Change" column in Table 3 shows the difference between the current year's SD value and the previous year's SD value. The double-difference value was obtained through subtracting change value of the control group from the change value of the intervention group, which represents the variation of the intervention group compared with the control group. In the intervention group, hospitalization expenditures decreased the most in the first year of DRG payment implementation, and decreased by 5,972 yuan more than the control group. The SD of the intervention group was smaller than that of the control group every year after DRG payment was implemented. 
Table 3

The SD and double-difference value of hospitalization expenses of patients with COPD

\begin{tabular}{|llllll|}
\hline \multirow{2}{*}{ Year } & \multicolumn{2}{l}{ Control Group } & \multicolumn{2}{l}{ Intervention Group } & \multirow{2}{*}{ Double-Difference } \\
\cline { 2 - 5 } & SD & Change & SD & Change & \\
\hline Before (FFS payment only) & & & & & \\
\hline 2010 & 17536.87 & - & 22798.05 & - & - \\
\hline 2011 & 15337.87 & -2199.00 & 17587.92 & -5210.13 & -3011.13 \\
\hline $\begin{array}{l}\text { After (DRG payment in pilot } \\
\text { hospitals) }\end{array}$ & & & & & \\
\hline 2012 & 10215.21 & -5122.67 & 6493.26 & -11094.66 & -5971.99 \\
\hline 2013 & 7037.40 & -3177.81 & 5778.24 & -715.02 & 2462.78 \\
\hline 2014 & 9009.47 & 1972.07 & 5789.14 & 10.91 & -1961.16 \\
\hline 2015 & 13955.59 & 4946.12 & 6538.47 & 749.32 & -4196.79 \\
\hline 2016 & 7037.71 & -6917.88 & 5681.75 & -856.71 & 6061.16 \\
\hline
\end{tabular}

Figure 1 shows the median, 75th percentile, 25th percentile, upper adjacent value, and lower adjacent value of the hospitalization expenditures for COPD patients from 2010 to 2016 . The length of the grey portion represents the IQR for each year. The red line represents the payment rate set for that DRG group. It can be seen that IQR is similar in the control and intervention groups before the reform. After implementing the DRG payment, the IQR of the intervention group decreased rapidly, and its value in each year was smaller than that of the control group. The difference between the lower adjacent value and upper adjacent value of the intervention group was much smaller than that of the control group, and there was a significant downward trend from 2010 to 2013. The control group did not see a significant reduction in the variation of hospitalization expenditures. After the reform, the median of hospitalization expenditures of the intervention group was basically stable and below the payment rate.

\subsection{Main results for patients with AMI}

Table 4 and Figure 2 show the changes in SD and IQR of hospitalization expenditures for AMI patients, respectively. As shown in Table 4, the SD of hospitalization expenditures decreased more in the intervention group than in the control group from 2012 to 2013. In 2012, the SD of hospitalization expenditures of the intervention group decreased by 2,483 yuan more than the control group, and further decreased by 4,112 yuan more than the control group in 2013. From 2014 to 2016, the SD of hospitalization expenditures of the intervention group increased, but the value was still less than that of the control group. After the implementation of DRG payment, the dispersion degree of hospitalization expenditures declined. 
Table 4

The SD and double-difference value of hospitalization expenses for patients with AMI

\begin{tabular}{|llllll|}
\hline Year & \multicolumn{2}{l}{ Matched Group } & \multicolumn{2}{l}{ Intervention Group } & \multicolumn{2}{l|}{ Double-Difference } \\
\cline { 2 - 5 } & SD & Change & SD & Change & \\
\hline Before (FFS payment only) & & & & & \\
\hline 2010 & 28310.83 & - & 26213.95 & - & - \\
\hline 2011 & 27514.44 & -796.39 & 24327.57 & -1886.38 & -1089.99 \\
\hline $\begin{array}{l}\text { After (DRG payment in pilot } \\
\text { hospitals) }\end{array}$ & & & & & \\
\hline 2012 & 25165.85 & -2348.59 & 19495.07 & -4832.50 & -2483.91 \\
\hline 2013 & 26146.35 & 980.50 & 16363.33 & -3131.73 & -4112.24 \\
\hline 2014 & 26776.27 & 629.92 & 18002.29 & 1638.96 & 1009.04 \\
\hline 2015 & 28379.07 & 1602.80 & 18546.57 & 544.28 & -1058.52 \\
\hline 2016 & 28120.67 & -258.40 & 20323.44 & 1776.87 & 2035.27 \\
\hline
\end{tabular}

As shown in Figure 2, after DRG payment was implemented, the hospitalization expenditures of the patients in the intervention group became increasingly concentrated. The IQR of the intervention group's hospitalization expenditures decreased significantly especially in 2012 and 2013. After the reform, the IQR value of the intervention group was smaller than that of the control group every year. From 2010 to 2016 , the median of hospitalization expenditures was less than the DRG payment rate in both groups, and the median of the intervention group was smaller than that of the control group.

\subsection{Main results of patient with cerebral infarction}

Table 5 and Figure 3 show changes in the SD and IQR of hospitalization expenditures for $\mathrm{Cl}$ patients. There were no patients with "cerebral ischaemic diseases with important complications and concomitant diseases group" in 2010, only data from 2011 to 2016 were available for Cl patients. After the reform, the SD of hospitalization expenditures for patients in the intervention group continued to decline, and decreased more in the intervention group than in the control group, except in 2014. The variation degree of hospitalization expenditures in the intervention group decreased year after year, and the SD in each year was less than that in the control group. 
Table 5

The SD and double-difference value of hospitalization expenses for patients with cerebral infarction

\begin{tabular}{|llllll|}
\hline \multirow{2}{*}{ Year } & \multicolumn{2}{l}{ Matched Group } & \multicolumn{2}{l}{ Intervention Group } & \multicolumn{2}{l|}{ Double-Difference } \\
\cline { 2 - 5 } & SD & Change & SD & Change & \\
\hline Before (FFS payment only) & & & & & \\
\hline 2011 & 15755.92 & - & 13571.37 & - & - \\
\hline $\begin{array}{l}\text { After (DRG payment in pilot } \\
\text { hospitals) }\end{array}$ & & & & & \\
\hline 2012 & 13612.52 & -2143.39 & 8583.88 & -4987.49 & -2844.09 \\
\hline 2013 & 16126.07 & 2513.55 & 8159.43 & -424.45 & -2938.00 \\
\hline 2014 & 11558.56 & -4567.51 & 6681.67 & -1477.76 & 3089.75 \\
\hline 2015 & 12238.64 & 680.08 & 6076.18 & -605.49 & -1285.56 \\
\hline 2016 & 14112.71 & 1874.07 & 5967.37 & -108.82 & -1982.89 \\
\hline
\end{tabular}

As shown in Figure 3, prior to the implementation of the policy, the IQR was similar for patients in the intervention and control groups. The variation in hospitalization expenditures was large in both groups. After the beginning of the policy, the IQR decreased significantly in the intervention group. Since 2013, the median of hospitalization expenditures was lower than the fixed payment rate of DRG payment. In the control group, the variance in hospitalization expenditures had no downward trend, and the median was always higher than the payment rate of DRG payment.

\section{Discussion}

It is widely recognized that DRG is one of the most advanced and scientific payment methods in the world. Under the implementation of DRG payment, hospitals can keep any savings but also bear any cost overruns (8). That is, the source of profit under FFS payment, such as drugs and medical materials, can become the source of costs under the DRG system (10). Therefore, financial risk is further transferred to the provider. In particular, when the hospitalization expenditures exceed the DRG payment rates, the provider has more initiative to reduce the overrun cost (7). Induced demand will be suppressed, which will help doctors implement standard treatment regimens in accordance with authoritative clinical guidelines. By analysing the trend of variation in hospitalization expenditures for patients with similar medical conditions, we can analyse whether DRG payment can contribute to the consistency of health services.

The study found that when there were no significant differences in age, gender, disease severity, or disease type, the variation of hospitalization expenditures and its changing trend with time were still different among patients with different payment methods. Compared with the patients under FFS payment, patients under DRG payment had less variation in hospitalization expenditures. The variation in 
most years showed a downward trend, and the decrease was larger in the intervention group than that in the control group. The median of hospitalization expenditures for the intervention group was lower than the payment rate, and the opposite was observed in the control group. This indicates that the policy has played a certain role in reducing hospitalization expenditures and their variation. In patients with the three disease types, the use of clinical resources was more consistent after DRG payment. Since key clinical decisions are made by doctors, it can be assumed that the reduction in the variation in hospitalization expenditures presented in the study is due to more consistent treatment behaviours by doctors. This supports the premise that DRG payment helps suppress the induced-demand of FFS payment and promotes a more standard treatment plan that is closer to clinical guidelines.

The study also found that patients with AMI had the least reduction in the variation of hospitalization expenditures before and after DRG payment. This may be explained as that the treatment regimen of AMI has been very mature before the implementation of the policy, and universal consensus with clinical guidelines has already been established, so doctors' behaviours have been regulated by a certain degree. Therefore, the effect of DRG payment on the variation in AMI hospitalization expenditures is not as obvious as that of other diseases. In addition, according to the analysis of hospitalization expenditures' composition, most expenditures in the internal medicine group belong to drug expenditures, while medical materials are the greatest source of expenditures in the surgical group. It can be inferred that in the management of DRG payment, the internal medicine group and the surgical group can make medical services more consistent by regulating the use of drugs and medical materials, respectively.

Previous studies have shown that compared with FFS, DRG payment has achieved shorter hospital stays (8), slower medical cost growth (11), better care processes (9), more efficient management $(12,13)$ and a more reasonable cost structure. Past experience shows that the variation in hospitalization expenditures will decrease after DRG payment implementation (6)which is largely consistent with the results of this study. Based on this effect, we tried to explore the main factors behind the decrease. The causes of variation in DRG internal resource utilization are complex. Obviously, one potential explanatory variable is the severity of the disease, but this is not the only variable or the most important one. The study showed that the average cost of the same procedure varied among surgeons (14), and the differences between doctors are even greater than those between hospitals (15). Therefore, doctors must be a primary control variable (16) in interpreting DRG internal resource changes. Studies have shown that changes in doctors' practice can reduce the variation in hospitalization expenditures more than the severity of illness (16). The decrease in hospitalization days was due more to changes in doctors' behaviour than to a decrease in disease severity $(5,6)$. This provides further support for the results of this study. After controlling for individual difference, the reduction in the variation in hospitalization expenditures is more likely due to a more consistent clinical practice among doctors.

\section{Limitations}

1. In this study, the impacts of doctors' behaviour on the reduction of variation in hospitalization expenditures were examined by controlling the individual differences of patients. However, the reduction 
in the variation of hospitalization expenditures is not the same as the regulation of doctors' practices. In the future, detailed measurement of doctors' practices should be conducted based on information such as specific treatment programs.

2. Although the results show a reduction in the variation of hospitalization expenditures, the consistency of hospitalization expenditures is not always equivalent to the standardization of health services. It remains to be analysed whether the clinical resources of patients are used in the most rational way and whether there is a risk of inadequate services affecting the quality of care. Future research should focus on which types of expenditures are most effective in improving quality and which types of expenditures represent waste. A complete list of necessary treatments should be established. The balance between reasonable cost control and reasonable diagnosis and treatment under DRG should be analysed.

\section{Conclusions}

Compared with FFS, DRG payment has resulted in a consistent trend in health expenditures for patients with similar conditions. It is suggested that the policy can encourage health service providers to actively control costs and promote more consistent behaviour among doctors. This is helpful in forming a dynamic control system for medical costs based on clinical pathway management, and it is a step towards achieving the goal of reducing costs and improving quality of care. However, the consistency of hospitalization expenditures is not equal to the standardization of health services. Hospitals should better understand the source of the decrease in the variation of hospitalization expenditures, give attention to the construction and implementation of clinical pathways, and set up corresponding evaluation systems based on diagnosis and treatment protocols, and finally promote the standardization of doctors' practices. These practices play an important role in achieving the goal of the DRG policy, which is a win-win situation for all three parties: health insurers, hospitals and patients.

\section{Abbreviations}

DRG: diagnosis-related group

COPD: chronic obstructive pulmonary disease

AMI: acute myocardial infarction

Cl: cerebral infarction

PSM: propensity-score-matched

FFS: fee for service

CCl: charlson comorbidity index

$\mathrm{CPI}$ : consumer price index 
SD: standard deviation

IQR: interquartile range

\section{Declarations}

\section{Ethics approval and consent to participate}

Ethical approval for this study was obtained from Peking University (IRB IRB00001052-18005).

All methods were carried out in accordance with relevant guidelines and regulations (declaration of Helsinki).

Informed consent was obtained from all subjects and/or their legal guardian(s).

\section{Consent for publication}

Not applicable

\section{Availability of data and materials}

The datasets generated and/or analyzed during the current study are not publicly available due to legal restrictions and confidential nature of the individual privacy but are available from the corresponding author on reasonable request.

\section{Competing interests}

The authors declare that they have no competing interests.

\section{Funding}

This work was supported by the National Natural Science Foundation of China(Grant Number: 72174007) and China Medical Board (Grant Number: 16-259).

\section{Authors' contributions}

WJ designed the study. XF was primarily responsible for data analysis and writing of the article. ZW, SH and ZC participated in the writing of the manuscript. All authors have read and approved the final draft. Each author believes that the manuscript represents honest work.

\section{Acknowledgements}

Not applicable.

\section{References}


1. Anderson GF, Chalkidou K. Spending on medical care: more is better? Jama. 2008;299(20):2444-5.

2. Fisher E, Skinner J. Comment on Silber et al.: Aggressive treatment styles and surgical outcomes. Health Serv Res. 2010;45(6 Pt 2):1893-902; discussion 908-11.

3. Huerta TR, Ford EW, Peterson LT, Brigham KH. Testing the hospital value proposition: an empirical analysis of efficiency and quality. Health Care Manage Rev. 2008;33(4):341-9.

4. lezzoni LI, Shwartz M, Ash AS, Mackiernan YD. Does severity explain differences in hospital length of stay for pneumonia patients? J Health Serv Res Policy. 1996;1(2):65-76.

5. Rodin D, Chien AT, Ellimoottil C, Nguyen PL, Kakani P, Mossanen M, et al. Physician and facility drivers of spending variation in locoregional prostate cancer. Cancer. 2020;126.

6. McMahon LF, Jr., Newbold R. Variation in resource use within diagnosis-related groups. The effect of severity of illness and physician practice. Med Care. 1986;24(5):388-97.

7. Yip W, Hsiao WC. The Chinese Health System At A Crossroads. Health Affairs. 2008;27(2):460-8.

8. Jian W, Lu M, Chan KY, Poon AN, Han W, Hu M, et al. Payment Reform Pilot In Beijing Hospitals Reduced Expenditures And Out-Of-Pocket Payments Per Admission. Health Aff (Millwood). 2015;34(10):1745-52.

9. Kahn KL, Rogers WH, Rubenstein LV, Sherwood MJ, Reinisch EJ, Keeler EB, et al. Measuring quality of care with explicit process criteria before and after implementation of the DRG-based prospective payment system. Jama. 1990;264(15):1969-73.

10. Herman AA. The effect of DRGs on termination-of-treatment issues. Perspect Healthc Risk Manage. 1991;11(4):19-25.

11. Kwak SH, Kim JH, Kim DH, Kim JM, Byeon HK, Kim WS, et al. Impact of the Korean Diagnosis-Related Groups payment system on the outcomes of adenotonsillectomy: A single center experience. Auris Nasus Larynx. 2018;45(3):504-7.

12. Palmer $G$, Reid $B$. Evaluation of the performance of diagnosis-related groups and similar casemix systems: methodological issues. Health Services Management Research An Official Journal of the Association of University Programs in Health Administration. 2001;14(2):71.

13. Ammar W. Hospital accreditation, reimbursement and case mix: links and insights for contractual systems. BMC Health Services Research. 2013;13.

14. Kazberouk A, Sagy I, Novack V, Mcguire K. Understanding the Extent and Drivers of Inter-physician Cost Variation for Spine Procedures. Spine. 2016;41(13):1111-7.

15. Tsugawa Y, Jha AK, Newhouse JP, Zaslavsky AM, Jena AB. Variation in Physician Spending and Association With Patient Outcomes. Jama Internal Medicine. 2017;177(5):675.

16. McMahon, Laurence, F., suffix:Jr., Newbold, Robert. Variation in Resource Use Within Diagnosisrelated Groups. Medical Care. 1986.

\section{Figures}



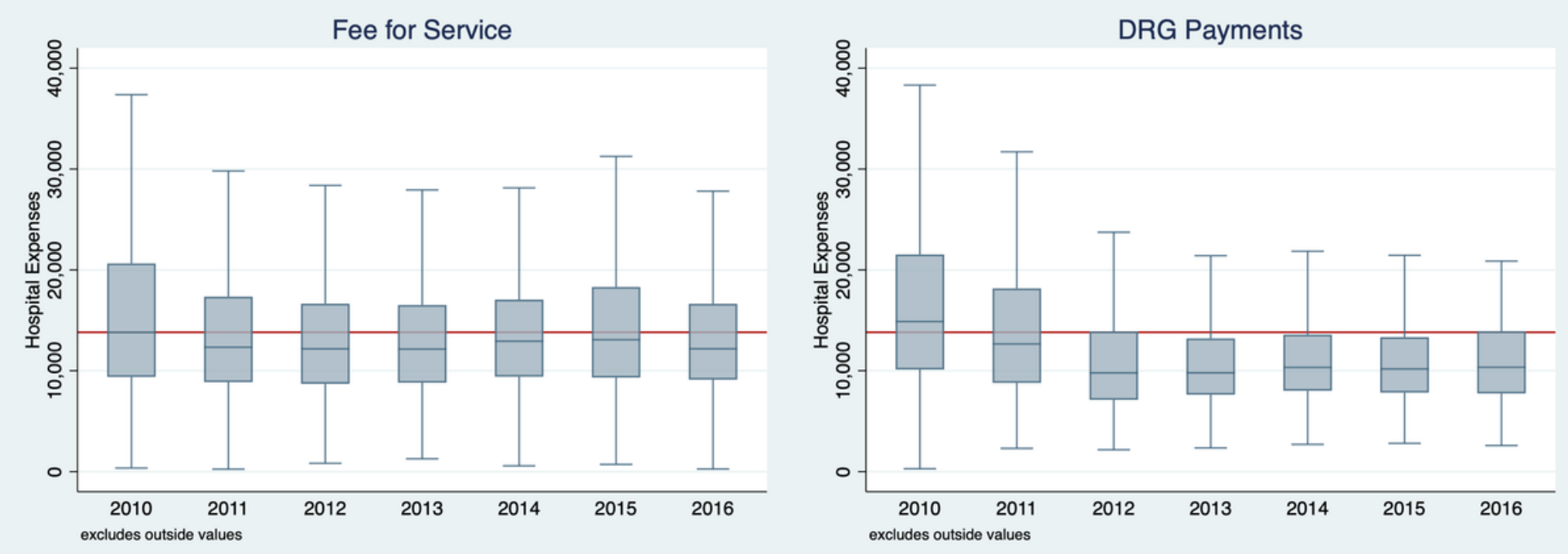

Figure 1

Box chart of hospitalization expenses of patients with COPD
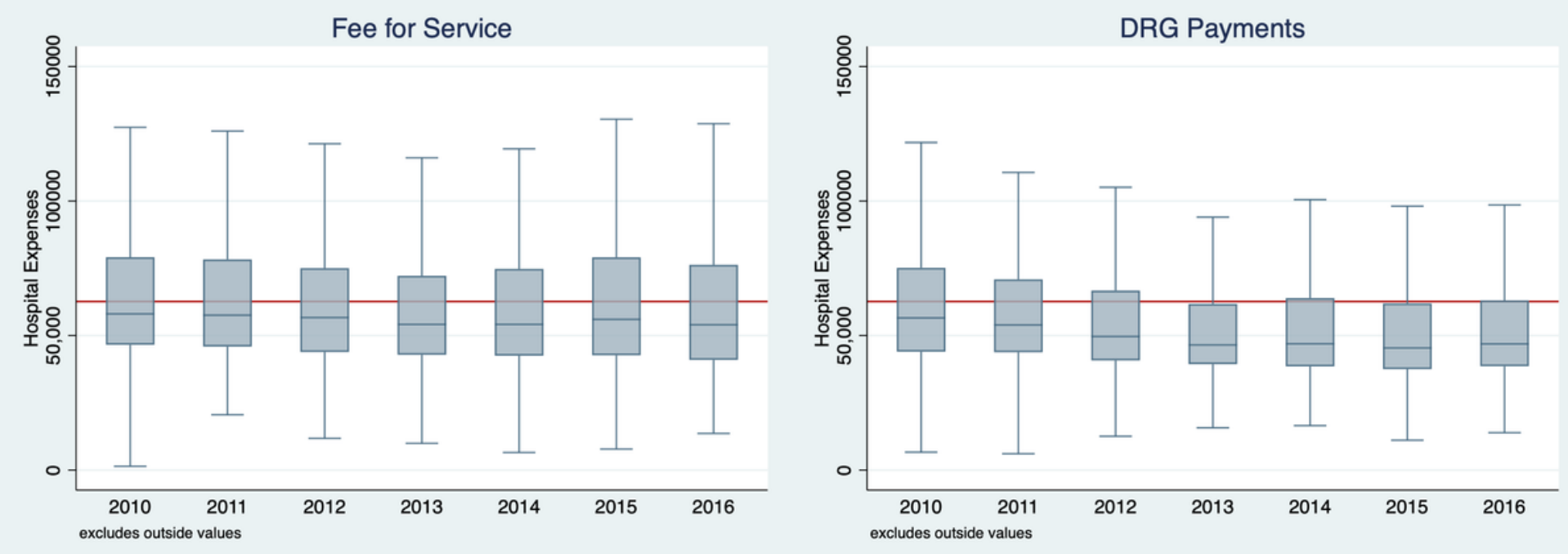

Figure 2

Box chart of hospitalization expenses for patients with AMI 

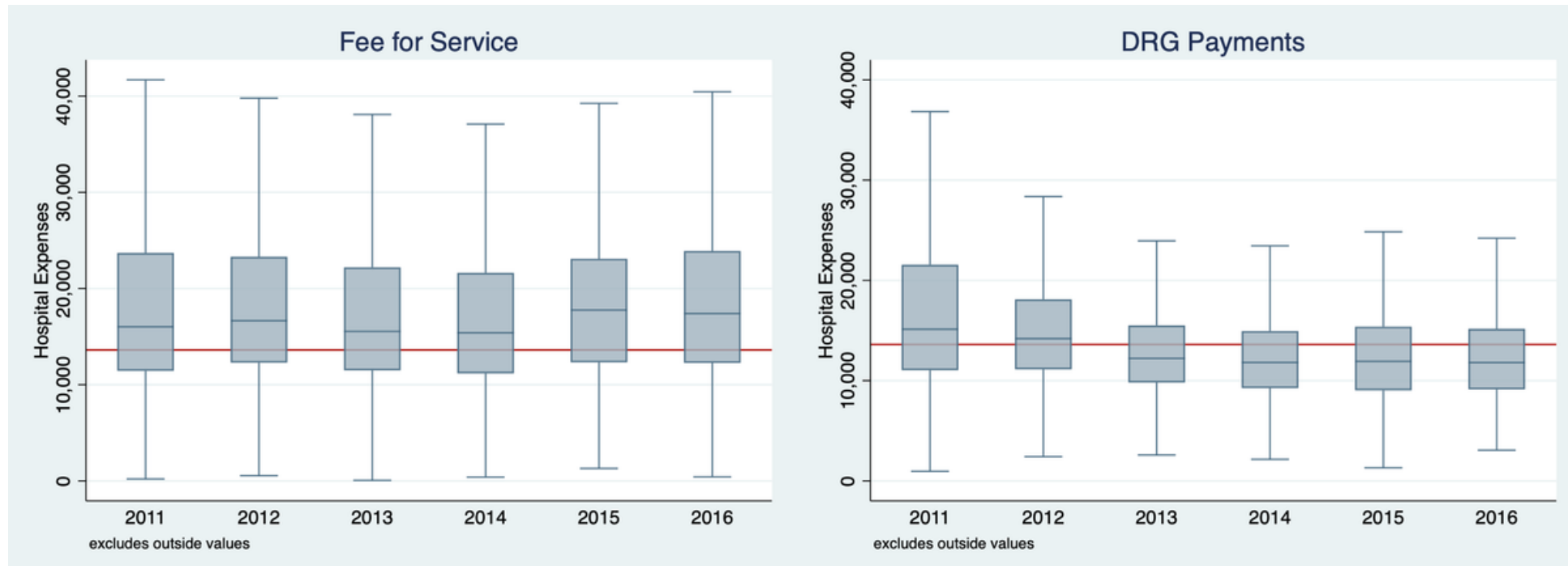

Figure 3

Box chart of hospitalization expenses of patients with cerebral infarction 